Research Article

\title{
New Strategies in the Code of Uncertainty and Sensitivity Analysis (CUSA) and Its Application in the Nuclear Reactor Calculation
}

\author{
Jiayu Du, Chen Hao (D), Ji Ma, Peijun Li, Xiaoyu Zhou, and Lixun Liu \\ Fundamental Science on Nuclear Safety and Simulation Technology Laboratory, Harbin Engineering University, \\ Harbin 150001, China \\ Correspondence should be addressed to Chen Hao; haochen.heu@163.com
}

Received 4 November 2019; Accepted 14 January 2020; Published 11 February 2020

Academic Editor: Tomasz Kozlowski

Copyright ( $\odot 2020$ Jiayu Du et al. This is an open access article distributed under the Creative Commons Attribution License, which permits unrestricted use, distribution, and reproduction in any medium, provided the original work is properly cited.

Best-Estimation Plus Uncertainty (BEPU) analysis method can provide more information to improve the reliability of calculation results than the safety analysis with conservative assumption. And the statistical sampling-based uncertainty and sensitivity analysis methods are widely used in practical applications of the multiphysics, multiscale coupling nuclear reactor system. In this paper, a novel and efficient sampling method for inputs with normal and uniform distributions is introduced and a systematic theory for uncertainty and sensitivity analysis is established based on the classical statistical theory. Then the Code of Uncertainty and Sensitivity Analysis (CUSA) is updated based on these new strategies. For applications, the explicit and implicit effects for resonance and nonresonance isotopes are studied in depth, and a simple $\mathrm{UO}_{2}$ pin cell is considered to examine the performance of CUSA and the total uncertainty and sensitivity analysis abilities. The numerical results indicate that the implicit sensitivity analysis model and the uncertainty quantification functions developed in CUSA are correct and can be used for sensitivity and uncertainty analysis in nuclear reactor calculations. Even more important, the LHS-SVDC is recommended to propagate the uncertainties in multigroup cross sections.

\section{Introduction}

Accurate estimation of key parameters of nuclear reactor is essential for both reactor simulation and safety analysis. Due to the fact that the nuclear reactor is a complex nonlinear multiphysics, multiscale coupling system, the high-fidelity simulations and modelling with full consideration of the coupling among neutronics, thermal hydraulics, fuel performance, and so on have now become the standard for modern evaluation of nuclear reactor system [1]. However, uncertainties inevitably propagate in the progress of nuclear reactor simulations $[2,3]$. The uncertainties of input parameters, manufacturing tolerance, approximation in calculation models, and so on naturally exist [4]. As research progressed and Best-Estimate (BE) methods matured, a move toward Best-Estimation Plus Uncertainty (BEPU) analysis occurred. BEPU can be used to quantify these uncertainties, and it provides more information to improve the reliability of calculation results than the safety analysis with conservative assumption. It is worthy to note that only the uncertainty of nuclear data is quantified by using the BEPU methods in this work.

For uncertainty quantification and propagation, two primary kinds of methods are widely used, e.g., the adjointbased uncertainty quantification and sensitivity analysis [5] and the statistical sampling-based uncertainty and sensitivity analysis method [6]. But for some practical applications, the adjoint-based uncertainty method is not going to work because of the inexistence of the solution of the adjoint equation or the difficulty in obtaining adjoint solutions. Although the statistical sampling-based uncertainty and sensitivity analysis method is to some extent time consuming, this method still has some significant advantages, such as less simplifications and approximations, and no limit on the system responses compared with the adjoint based method. Hence, the statistical sampling-based uncertainty and sensitivity analysis methods are widely used in practical applications of the multiphysics, multiscale coupling nuclear 
reactor system such as assembly burnup [7]; power distribution [8]; decay heat [9]; control rod worth [10]; filling fraction of pebble bed [11]. Moreover, some codes based on this method also have been developed for uncertainty and sensitivity analysis, such as SUSA [12], XSUSA [13], Dakota [14], and CUSA [15].

The CUSA (the Code for Uncertainty and Sensitivity Analysis) was originally developed for uncertainty and sensitivity analysis of filling fraction of pebble bed and uranium loading of fuel pebble in pebble bed high temperature reactor (HTR-PM). The initial version only has some simple sampling and statistical functions based on the Simple Random Sampling (SRS) method, Latin Hypercube Sampling (LHS) method, K-S test, and basic statistical theory. And it is tightly coupled with the simulation codes for HTR-PM. As for the implementation progress of the statistical sampling-based uncertainty and sensitivity analysis, a set of input parameters should be generated firstly by random sampling according to their distribution and covariance information. Then these sets of input parameters will be sent into the same calculation to produce a set of outputs. At last, the statistical theory is applied to quantify the uncertainty of outputs propagated from inputs. So using the statistical sampling method to perform uncertainty and sensitivity analysis, two key technical problems should be considered reasonably and efficiently. First, efficient sampling methods should be studied to obtain a reasonable input sample space based on the covariance and distribution information. Second, a systematic theory analysis for uncertainty quantification should be conducted, which can be used to quantify the uncertainty of output and its associated error bar or confidence interval under a specific size of sample space.

In this paper, a novel and efficient sampling method for inputs with normal and uniform distributions is introduced, which is based on the well-known LHS method and Singular Value Decomposition (SVD). The efficient sampling method based on the SVD for high-dimensional covariance matrix is also proposed. Then, a systematic theory for uncertainty and sensitivity analysis is established based on the classical statistical theory, which can be used to quantify the distribution type of outputs, uncertainty, and its associated error bar under a specific size of samples. Then a new version of CUSA has been developed and some new functions based on the innovative efficient sampling methods, correlation control techniques, and uncertainty quantification methods have been updated. At the same time, a user-friendly interface for CUSA has been developed and the interface also serves as an integrated platform for controlling all the function modules and coupling with the executable version of other simulation codes. Even more important, the CUSA code can be used for uncertainty and sensitivity analysis for a wide range of applications, such as uncertainty analysis for reactor physics, thermal hydraulics calculations, and safety analysis.

For applications, the uncertainties of effective resonance self-shielding cross sections and total uncertainty of eigenvalue propagated from multigroup microscopic cross sections are quantified by using the CUSA and a homedeveloped resonance calculation code. Even more important, the explicit and implicit effects for resonance and nonresonance isotopes are fully considered. And the total uncertainty analysis can be conducted not only for the integral cross sections, including $\sigma_{a}, \sigma_{s}$, and $\sigma_{t}$, but also for the basic cross sections, such as $\sigma_{\text {elas }}, \sigma_{\text {inel }}, \sigma_{f}$, and $\sigma_{\gamma}$. In this way, the multigroup microscopic cross section uncertainties can be propagated to the neutronics responses completely. In this paper, the uncertainty quantified by CUSA and "Sandwich Rules" is compared based on a simple $\mathrm{UO}_{2}$ pin cell in order to examine the performance and ability of CUSA. Meanwhile, it should be noted that this paper aims to introduce the good sampling and statistical methods of CUSA and apply it to sensitivity and uncertainty analysis of effective resonance self-shielding cross sections and eigenvalue.

In the following sections, the details of methodologies and basic functions of CUSA are described and then in Section 3, the methods of uncertainty and sensitivity analysis for effective resonance self-shielding cross sections and eigenvalue by applying CUSA are discussed. Section 4 shows a detailed analysis of the origin sensitivity and uncertainty information and the total uncertainty results for $\mathrm{UO}_{2}$ pin cell. At last, conclusions drawn from this work are given in Section 5 .

\section{Methodologies and Basic Functions of CUSA}

\subsection{Efficient Sampling Methods}

\subsubsection{Basic Sampling Theory for Normal and Uniform} Distributions. The basis of the implemented statistical sampling-based uncertainty and sensitivity analysis method is to treat all uncertain inputs as dependent random variables by sampling them according to a multivariate probability density function (PDF). The mean and variance/covariance of the variable PDFs must be known to perform the statistical sampling uncertainty propagation. And an $n \times n$ covariance matrix is used to describe the uncertainty associated with each element of the input vector and the correlations that exist between elements. Rather than generating new vectors directly from their PDFs, an alternative approach is to generate vectors of perturbation factors that when multiplied by the reference input vector will produce a population of vectors, whose mean value is equal to the reference and whose elements have dependencies.

For variables obeying the normal distribution, the normally distributed dependent random samples can be generated as shown as follows [16]:

$$
X=A Z+\mu
$$

where $Z$ is a normally distributed independent vector, whose mean value is equal to 0 and standard deviation is $1 . \mu$ is the mean vector of input variables and the matrix $A$ can be generated based on the Singular Value Decomposition of the relative covariance matrix $\Sigma$ : 


$$
\Sigma=U S V^{T}=U \sqrt{S}(\sqrt{S})^{T} U^{T}=U \sqrt{S}(U \sqrt{S})^{T}=A A^{T} .
$$

But for uniformly distributed variables, the approach for normally distributed variables mentioned above will fail; then a new two-step transformation approach based on Singular Value Decomposition is proposed to generate the uniformly distributed dependent random samples. The basic idea of this two-step method is illustrated in Figure 1.

As shown in Figure 1, a standard normally distributed independent vector $Z$ should be generated, and then the linear transformation technique is applied to obtain the normally distributed dependent random samples $Y$ :

$$
Y=A Z,
$$

where $A$ is generated by performing SVD of the correlation coefficient matrix $C_{Y}$ of the random elements of the vector $Y$ :

$$
C_{Y}=U S V^{T}=U \sqrt{S}(U \sqrt{S})^{T}=A A^{T} .
$$

Here, the correlation coefficient matrix of $Y$ is not known. But the correlation coefficient matrix $C_{X}$ of $X$ is known as the basic input information. So the key technique is to determine the relationship between the correlation coefficient of different random elements of $X$ and $Y$. Based on the rigorous mathematical derivation, the relationship can be established by [17]

$$
\rho_{y_{i} y_{j}}=2 \sin \left(\frac{\pi}{6} \rho_{x_{i} x_{j}}\right) .
$$

As we know, the cumulative probability density function of $Y$ is consistent with the cumulative probability density function of $X$ by nonlinear transformation. And the cumulative probability density function of the standard normally distributed samples can be represented by the error function, as shown in

$$
\int_{-\infty}^{y_{i}} f_{Y_{i}}\left(y_{i}\right) \mathrm{d} y_{i}=F_{Y_{i}}\left(y_{i}\right)=\frac{1}{2}\left[1+\operatorname{erf}\left(\frac{y_{i}}{\sqrt{2}}\right)\right] .
$$

In this way, the desired uniformly distributed dependent random samples can be generated as shown as follows:

$$
x_{i}=F_{X_{i}}^{-1}\left[\frac{1}{2}\left(1+\operatorname{erf}\left(\frac{y_{i}}{\sqrt{2}}\right)\right)\right] .
$$

2.1.2. Correlation Control Method. As discussed above, $Z$ is a vector including $n X$ independent normally distributed random variables, which obeys the multivariate standard normal distribution $N_{n X}(0, I)$. So the success of the sampling is to generate an excellent sample space $Z_{s}$ of $Z$ firstly. And the samples should meet the following two conditions: (1) the correlation matrix associated with $Z_{s}$ is very close to the $n X \times n X$ identity matrix; (2) the mean vector is a zero vector. Normally, the SRS or LHS method is applied to generate the desired sample space $Z_{s}$ and the covariance matrix of $Z_{s}$ is denoted as $\Sigma_{s}$. However, $\Sigma_{s}$ always does not meet the above two conditions. Especially, when the sample size is small, a strong correlation will occur, which will lead to a distortion of the sample space and have a potentially large impact on the uncertainty propagation. Therefore, efficient sampling methods of fully considering the correlation information between different elements should be studied to obtain a reasonable input sample space based on the covariance and distribution information. In our work, a novel and efficient sampling method for inputs with normal and uniform distributions is proposed, which is based on the well-known LHS or SRS or Importance Sampling (IS) method combined with SVD technique [18]. Then, a desired sample space $Z_{s}^{*}$ can be obtained as follows [18]:

$$
\Sigma_{s}=\frac{1}{n} Z_{s}^{T} Z_{s}-\frac{1}{n^{2}} Z_{s}^{T} H Z=U S V^{T},
$$

where $H$ is an $n \times n$ full matrix with the element of $1 . S$ is a diagonal matrix and the diagonal elements are the singular value of $\Sigma_{s}$. And $\Sigma_{s}$ is the covariance matrix of $Z_{s}$, which is a symmetric matrix. So the matrix $U$ is the same as $V$; then (8) can be rewritten as

$$
U^{T} \Sigma_{s} U=S
$$

Further, two new diagonal matrices denoted as $E$ and $D$ are constructed, in which, $E \in R^{n X \times n X}, e_{i, i}=\sum_{s, i, i}, D \in R^{n X \times n X}$, and $d_{i, i}=\sqrt{s_{i, i} / \Sigma_{s, i, i}}$.

Then, a new formula is obtained as

$$
\left(D^{-1}\right)^{T} S D^{-1}=E \text {. }
$$

Combining (10) with (9) and inserting this two formulae into (8), we can get

$$
\frac{1}{n}\left(Z_{s} U D^{-1}\right)^{T} Z_{s} U D^{-1}-\frac{1}{n^{2}}\left(Z_{s} U D^{-1}\right)^{T} H Z_{s} U D^{-1}=E .
$$

Based on (11), we know that the covariance matrix of the sample space $Z_{s}^{*}=Z_{s} U D^{-1}$ is a unit diagonal matrix, which indicates that the parameters in the sample space are completely independent. So, a new independent sample space $Z_{s}^{*}$ is obtained, which strictly obeys the multivariate standard normal distribution $N_{n X}(0, I)$.

\subsubsection{Sampling Method for High-Dimensional Covariance} Matrix. In some practical applications, the $n \times n$ covariance matrix for representing the uncertainty and correlation information of all uncertain inputs is very large, probably thousands or more, and we call it high-dimensional covariance matrix. For example, the size $n$ of the global covariance matrix for the multigroup microscopic cross sections is very large if fine energy group and all nuclides are considered, although most of the off-diagonal elements are zero. If the sample size $n S$ is bigger than or equal to the matrix size $n$, the methods mentioned above can be used efficiently. But very often the physical calculations become the bottleneck and require the most computational effort. In fact, a relative small sample size, which can represent most of the uncertainty and correlation information, is useful for practical applications. If the $n S$ is smaller than the matrix size 


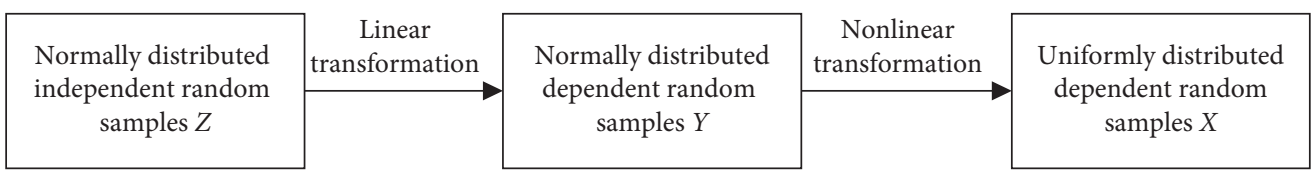

Figure 1: The basic ides of the two-step transformation approach.

$n$, the covariance matrix of the samples of $Z$ is a singular matrix. Therefore, the correlation control technique based on the SVD fails and leads to a distorted uncertainty propagation. To solve this problem, a reduced order sampling technique based on principal component analysis is proposed.

First, the SVD is performed on the $n \times n$ covariance matrix of the uncertain inputs. Then, the principal component analysis is performed on the singular value matrix $S$, and the main $n S V$ singular values are selected to construct a new singular value matrix $S_{1}$; then

$$
\begin{aligned}
\Sigma & =U S U^{T}=\left[\begin{array}{ll}
U_{1} & U_{2}
\end{array}\right]\left[\begin{array}{cc}
S_{1} & 0 \\
0 & S_{2}
\end{array}\right]\left[\begin{array}{ll}
U_{1} & U_{2}
\end{array}\right]^{T} \\
& =U_{1} S_{1} U_{1}{ }^{T}+U_{2} S_{2} U_{2}{ }^{T},
\end{aligned}
$$

where the matrix $U_{2} S_{2} U_{2}{ }^{T}$ can be ignored if enough singular values are selected. In this way, the $n \times n$ covariance matrix of the uncertain inputs can be approximated to the following:

$$
\Sigma=A A^{T} \approx U_{1} S_{1} U_{1}^{T}=U_{1} \sqrt{S_{1}}\left(U_{1} \sqrt{S_{1}}\right)^{T} .
$$

After the principal component analysis, the original covariance matrix can be approximated to a high degree of accuracy with very few components. In this way, a relatively small sample size, such as 100 , will be enough. In addition to the computational gains, the correlation control method can be no longer limited in the high-dimensional matrix applications.

\subsection{Uncertainty and Sensitivity Analysis Methods Based on} Statistical Theory. For uncertainty quantification, the Kolmogorov-Smirnov (K-S) test [19] is chosen to compare whether the distribution of a population sample is significantly different from the overall distribution and judge the sample distribution type, such as normal distribution, uniform distribution, and chi-square distribution. When the significance level is less than the given value (usually 0.05), the sample space is considered to have a significant difference from the population. Once the distribution type of the samples of outputs is determined, the typical statistical theory can be used to quantify the uncertainty information of the output, such as the mean value, standard deviation, and coefficient of variation. Here, the coefficient of variation is the ratio between the standard deviation and the mean in a sampled population, which represents the relative uncertainty.

However, the statistic itself has uncertainty and the standard error is usually used to represent this uncertainty. A standard error of a statistic is the standard deviation of the statistic. And an error bar is, in a plot, a line which is centered at the estimate with length that is double the standard error. It is well known that the error bar is a function of the number of samples and that accurate uncertainty estimates with narrow error bar will be observed for large sample sets. So a systematic uncertainty analysis should not only give the basic statistical information of outputs but also provide its associated error bar information under a certain sample size.

For the mean value, regardless of the type of distribution, as long as the sample size $n S$ is large, normally larger than 20, the standard error $\sigma_{\bar{O}}$ of the mean $\bar{O}$ can be quantified by [20]

$$
\sigma_{\bar{O}}=\frac{\sigma}{\sqrt{n S}}
$$

where $\sigma$ is the standard deviation of the output population.

For standard deviation, the standard error is different for different distribution types. In the case of large samples $(n S \geq 20)$, the associated standard error for the normal distribution and uniform distribution can be quantified by using (15a) [21] and (15b) [22], respectively:

$$
\begin{aligned}
& \sigma_{\sigma}=\frac{\sigma}{\sqrt{2(n S-1)}} \\
& \sigma_{\sigma}=\sqrt{\frac{2(n S-1)}{n S+2}} \frac{\sigma}{n S+1} .
\end{aligned}
$$

The absolute value of the coefficient of variation is usually termed as the relative standard deviation (RSD), and it represents the relative uncertainty in our research. In the case of large samples $(n S \geq 20)$, the standard error of the coefficient of variation for the normally distributed outputs can be determined by

$$
\sigma_{R S D}=\sqrt{\frac{C^{4}}{n S}+\frac{C^{2}}{2 n S}}
$$

where $C$ represents the coefficient of variation of the output population.

But for the uniformly distributed outputs, the standard error of the coefficient of variation is conservatively estimated by following equation in our present work:

$$
\begin{aligned}
\sigma_{R S D}= & \frac{C+\sqrt{2(n S-1) / n S+2}(C / n S+1)}{2(1-(C / \sqrt{n S}))} \\
& -\frac{C-\sqrt{2(n S-1) / n S+2}(C / n S+1)}{2(1+(C / \sqrt{n S}))} .
\end{aligned}
$$

It should be noted that the confidence interval of the statistic can also be used to represent the statistic itself uncertainty due to statistical process. In our work, the 
confidence interval with a certain confidence can also be quantified for the mean, standard deviation, and the coefficient of variation.

On the other hand, the study of how output uncertainty can be apportioned to the different input sources, which is known as sensitivity analysis, is an important part to the uncertainty quantification. Since the sensitivity analysis can be used to identify where a reduction of uncertainty should be performed, it has the greatest benefit for the reduction of the total uncertainty. For uncertainty and sensitivity analysis based on statistical theory, the sensitivity information cannot be obtained directly. In this paper, we estimate the sensitivity coefficients of effective self-shielding cross sections and eigenvalue to multigroup microscopic cross sections, which have a strong linear relationship. The direct perturbation method is a practical method for local sensitivity analysis, where the output variable is only changing due to a direct perturbation from a specific parameter. It is worth noting that when the two parameters do not obey a linear relationship, the direct perturbation method will not be applicable. In addition to local sensitivity analysis, global sensitivity analysis based on variance distribution is also a good method to estimate the sensitivity coefficients of nuclear data, but it is not used in this paper [23,24].

At the same time, the correlation analysis, which usually calculates the Kendall, Pearson, and Spearman correlation coefficients, is also used to perform sensitivity analysis to quantify the influence of the specific input parameter on the output.

\subsection{Main Functions and Interface Design}

2.3.1. Overview of Basic Functions of CUSA. Based on the main methods discussed above, a new version of CUSA has been developed and some new functions, such as efficient sampling, statistical analysis, standardized input, code coupling, and plot, have also been developed by using $\mathrm{C}$ language on windows operation system. An overview of basic functions and coupling relationship between different modules is illustrated in Figure 2.

For the standardized input module, the initial information of uncertainty sources should be first inputted to the CUSA, including mean value, the relative covariance information, and some basic parameters and description of inputs. And there are two ways for input, directly reading from the input files and inputting through interface manually. However, a specific format of input data needs to be provided, while there is no limitation by using the interface. For nuclear data uncertainty propagation and quantification by using CUSA, an important function of transforming nuclear cross-section covariance matrix in multigroup form into users' group structures is implemented based on our previous research [25]. By using this function, users only need to input the desired energy group information to obtain the relative covariance matrix in users' group structures, which is an easy way and recommended. Of course, users can input all the relative covariance information by using interface or input files.
According to the innovative efficient sampling methods and correlation control techniques, three fundamental sampling methods, including SRS, IS, and LHS, can be used to generate desired sample spaces. Once the correlation control techniques are taken into account, total 9 sampling methods can be used and the LHS-SVDC is recommended to generate an excellent sample space with a relatively small sample size, normally about 100 , for either normally distributed or uniformly distributed input parameters. For other distribution types, the SRS method is used to generate the desired sample space. All these functions can be easily applied by using the CUSA interface.

The statistical analysis module consists of three parts, K-S test, statistical calculation, and sensitivity analysis and also has two essential applications in CUSA. The first application is to verify the sample space, such as the mean value, relative standard deviation, correlation information, and distribution type. Second, and more important, this function is especially designed for uncertainty and sensitivity analysis. Uncertainty quantification (error bar and confidence interval), distribution type estimation, direct sensitivity analysis, and correlation analysis are all conducted by using this module.

There are two coupling strategies with other simulation codes, directly coupling with the dynamic-link library (DLL) or reading and generating the specified files for simulation codes. If the DLL of other simulation codes is coupled with CUSA, an important parallel calculation function can be used and the number of calculations can also be controlled.

At last, and more important, a user-friendly humancomputer interaction platform of CUSA is developed by using QT language. Based on this interface, all functions of CUSA can be performed. The initial and operation interfaces are shown in Figure 3. At the same time, the logo of CUSA is also designed for further application and development.

\section{Sensitivity and Uncertainty Analysis by Applying CUSA}

3.1. Sensitivity and Uncertainty Analysis of Effective Resonance Self-Shielding Cross Sections. For the application of CUSA, the uncertainties of effective resonance self-shielding cross sections propagated from multigroup microscopic cross sections through resonance calculation are quantified firstly. In this work, a home-developed resonance module based on subgroup method is applied for resonance calculation. It should be noted that the effective resonance self-shielding cross sections are related to the calculation of subgroup parameters and subgroup flux in the subgroup resonance calculation. For nonresonant nuclides, the uncertainty of the multigroup microscopic cross sections will only be propagated to the subgroup flux and further propagated to the final calculated effective resonance self-shielding cross sections. But for resonant nuclides, the uncertainty of the multigroup microscopic cross sections will be propagated to both the subgroup parameters and flux. So uncertainties of both nonresonant and resonant nuclides should be taken into account when quantifying the uncertainty of effective resonance self-shielding cross sections. In this way, the 


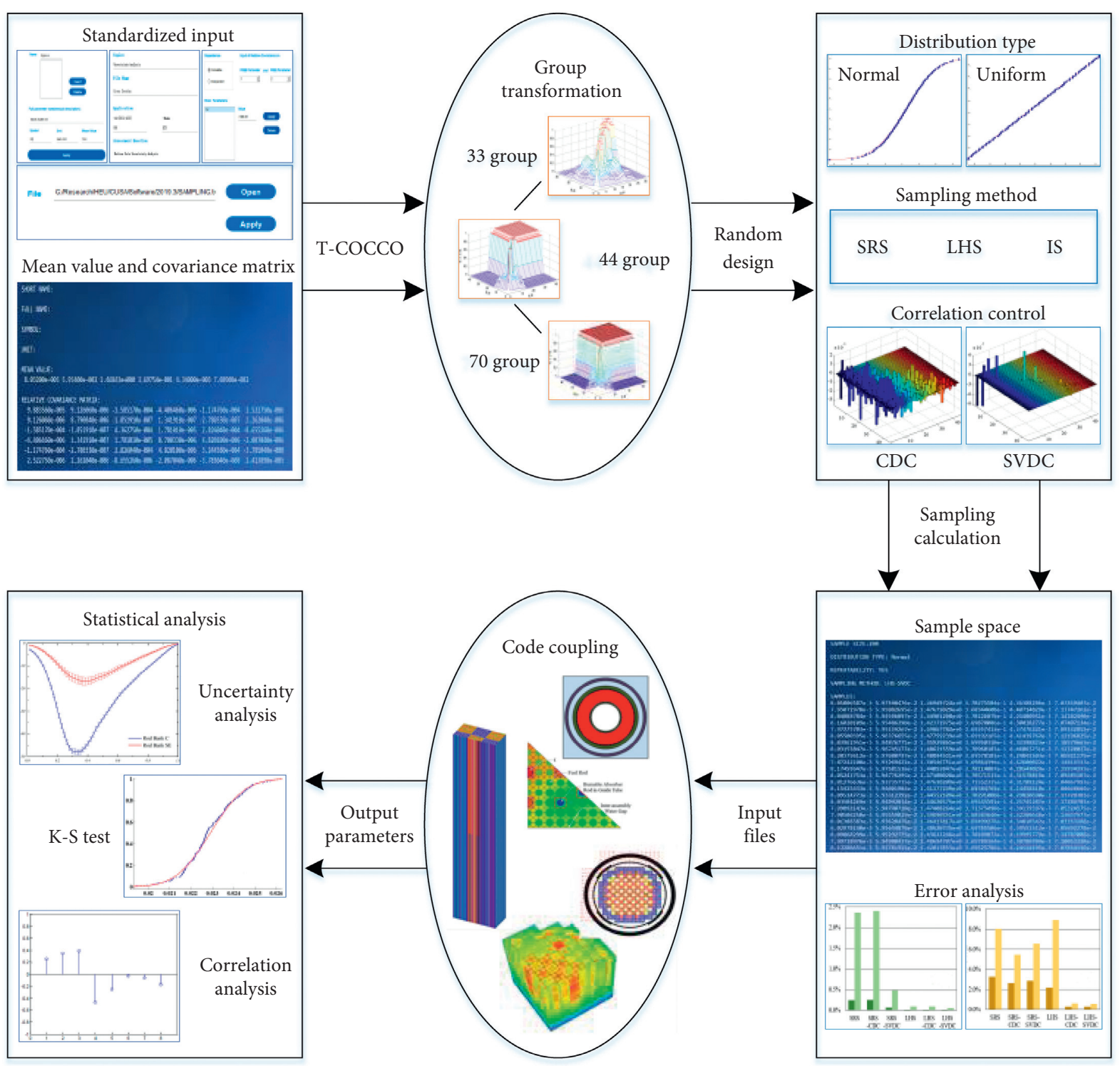

FIgURE 2: The function diagram of CUSA.

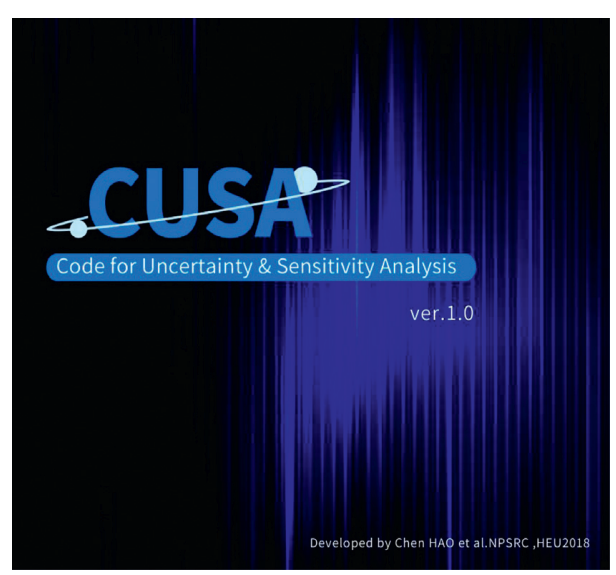

(a)

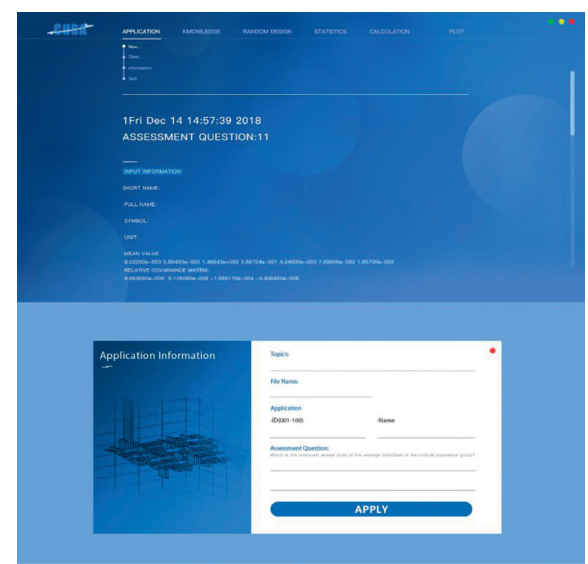

(b)

Figure 3: Initial and operation interface of CUSA. 
effects of microscopic cross sections on the effective resonance self-shielding cross sections, which are defined as the implicit effects, are fully considered.

At the same time, the cross-section consistency rules should be established in the multigroup microscopic crosssection perturbation model. As a WIMS 69-energy group based library is used in this work, for the resonance energy group, the absorption cross section is completely composed of the neutron capture cross section and fission reaction cross section, without the $(n, 2 n),(n, 3 n)$, and $(n, p)$ reactions. And the scattering cross section is completely composed of elastic scattering cross section. In this way, the following equations are applied as simplified consistency rules to keep the cross sections balanced:

$$
\begin{aligned}
& \sigma_{a}=\sigma_{(n, \gamma)}+\sigma_{f}, \\
& \sigma_{s}=\sigma_{\text {elastic }}
\end{aligned}
$$

The direct perturbation function built in CUSA is applied firstly to quantify the implicit sensitivity coefficients, which represent the indirect impact of multigroup microscopic cross sections on the response through the resonance self-shielding calculation. When a parameter perturbation is small enough, the change of the effective resonance selfshielding cross section can be expressed through the linear relationship. Then the sensitivity coefficient, which indicates the relative percent change of the effective resonance selfshielding cross section when the multigroup microscopic cross sections are perturbed with a relative value of $1 \%$, can be defined as

$$
S_{\sigma_{x, g}, \alpha_{g}}^{\operatorname{imp}}=\frac{\delta \sigma_{x, g} / \sigma_{x, g}}{\delta \alpha_{g} / \alpha_{g}}
$$

where $\alpha_{g}$ stands for the $g$ th group microscopic cross section of a particular nuclide and $\sigma_{x, g}$ stands for the effective resonance self-shielding cross section of the reaction type $x . \delta$ represents the perturbation of cross sections; in this work it is $1 \%$.

For uncertainty quantification, a relative covariance matrix is needed, which contains the uncertainty and correlation information for different nuclear reactions. And this matrix should be in the same energy group structure as the unique multigroup cross-section library used in the sensitivity analysis. This relative covariance matrix can be collapsed by a flux weighting directly from the evaluated data files such as ENDF to the user's group structure by using NJOY [26]. Although this way is more mathematically rigorous, the process is relatively complicated and the covariance information needs further judgements and comprehensive evaluations. Alternatively, an existing evaluated multigroup covariance matrix, such as ZZ-SCALE6.0/ COVA-44G library [27], can be transformed into a new energy group structure based on a flat-flux approximation when the widths of the energy groups are not drastically different. In this work, the module T-COCCO built in CUSA is applied to transform the covariance matrix in 44-energy group form into a 69-energy group structure to provide basic uncertainty information of multigroup cross sections.
Then efficient sampling and statistical analysis modules are used to quantify uncertainties of effective resonance selfshielding cross sections propagated from multigroup microscopic cross sections. At the same time, the "Sandwich" rule is also used to quantify the uncertainty based on the calculated implicit sensitivity coefficients for further comparison. As illustrated in Figure 4, the basic idea of quantifying uncertainties of the effective resonance self-shielding cross sections by using CUSA is summarized as the following steps:

(1) The original relative covariance matrix in 44-energy group and the basic information of the WIMS 69 energy group-based library, such as the energy group structure and mean of each cross section, are inputted to CUSA system.

(2) A relative covariance matrix in 69-energy group structure is generated by using T-COCCO to provide basic uncertainty information.

(3) Determine the sample size $n S$ and the efficient sampling module is called to generate a desired sample space for the multigroup microscopic cross sections, which will generate $n S$ perturbed multigroup cross-section libraries. Here, the cross-section consistency rules are applied to keep the cross sections balanced.

(4) The resonance calculation module based on subgroup method is coupled with CUSA by using the DLL file. Based on the perturbed libraries, the resonance calculations will be performed $n S$ times to generate $n S$ effective resonance self-shielding cross-section libraries.

(5) The statistical analysis module is then used to quantify the uncertainty of the effective resonance self-shielding cross sections and their associated error bar with the given sample size.

3.2. Sensitivity and Uncertainty Analysis of Eigenvalue by Applying CUSA. In order to extend the implicit sensitivity analytical ability of CUSA to a wider application and further to investigate its impact on the uncertainty results, the eigenvalue total sensitivity and uncertainty analysis is performed in our work. In addition, since the sensitivity analysis of the resonance energy groups is much more complicated than the sensitivity analysis of fast and thermal energy groups, which do not contain implicit sensitivity analysis, this paper is only focused on the sensitivity and uncertainty analysis of resonance energy groups.

The total sensitivity coefficient of eigenvalue should consist of two parts, explicit sensitivity coefficient and implicit sensitivity coefficient. If the perturbation of $g$ th group microscopic cross section only affects the corresponding macroscopic cross section and further affects the eigenvalue through transport calculations, this associated sensitivity coefficient is defined as explicit sensitivity coefficient, which can be calculated as

$$
S_{k_{\mathrm{eff}}, \alpha_{g}}^{\exp }=\frac{\delta k_{\mathrm{eff}} / k_{\mathrm{eff}}}{\delta \alpha_{g} / \alpha_{g}} .
$$




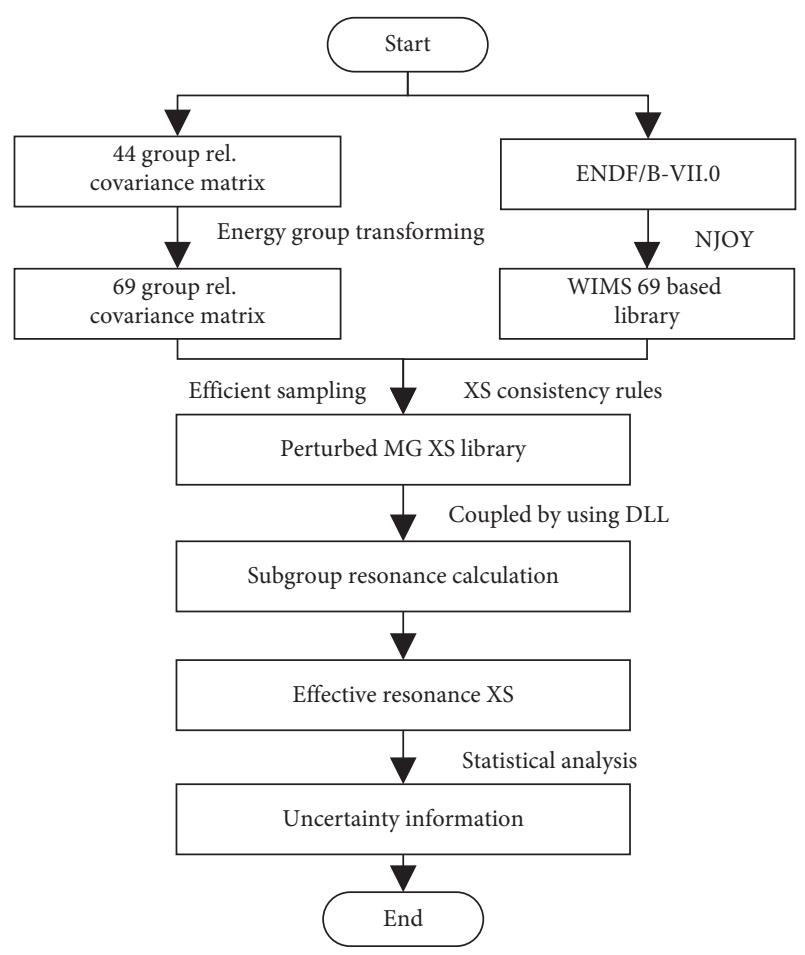

FIGURE 4: Flowchart of uncertainty analysis of effective resonance self-shielding XS by CUSA.

As mentioned in Section 3.1, the perturbation of the microscopic cross section has a direct effect on the effective resonance self-shielding cross sections first and further affects the macroscopic cross sections. At last, this effect will propagate to the final calculated eigenvalue through transport calculations. In this case, the sensitivity coefficient of eigenvalue to the multigroup microscopic cross section is considered as implicit sensitivity coefficient, which can be quantified by using the following equation:

$$
S_{k_{\text {eff }}, \alpha_{g}}^{\mathrm{imp}}=\sum_{j, x} \frac{\delta k_{\text {eff }} / k_{\text {eff }}}{\delta \sigma_{x, g}^{j} / \sigma_{x, g}^{j}} \frac{\delta \sigma_{x, g}^{j} / \sigma_{x, g}^{j}}{\delta \alpha_{g} / \alpha_{g}}=\sum_{j, x} S_{k_{\text {eff }}, \sigma_{x, g}^{j}}^{\exp } S_{\sigma_{x, g}^{j}, \alpha_{g}}^{\mathrm{imp}},
$$

where $j$ indicates resonant nuclides and $x$ is the indicator of the reaction type of effective resonance self-shielding cross sections. The first term of the implicit sensitivity coefficient is the sensitivity coefficient of the $k_{\text {eff }}$ to the effective resonance self-shielding cross sections, which is a kind of explicit sensitivity coefficient. The second term of the implicit sensitivity coefficient is the sensitivity coefficient of the effective resonance self-shielding cross sections to the multigroup micro cross sections, the details can be found in Section 3.1. Then, the total sensitivity coefficient can be calculated as

$$
S_{k_{\text {eff }}, \alpha_{g}}^{\text {tot }}=S_{k_{\text {eff }}, \alpha_{g}}^{\exp }+S_{k_{\text {eff }}, \alpha_{g}}^{\mathrm{imp}} .
$$

It should be noted that the calculations of total sensitivity coefficient for the resonant nuclides and nonresonant nuclides are slightly different. For the resonant nuclides, the explicit sensitivity coefficient term should be considered as zero. This is due to the fact that the explicit effect is already considered when calculating the implicit sensitivity coefficients for resonant nuclides. But for the nonresonant nuclides, since there is no effective resonance self-shielding cross section associated with its own multigroup microscopic cross section, the explicit effect is not considered in the process of quantifying its implicit effect on the effective resonance self-shielding cross sections of resonant nuclides.

The same as the uncertainty quantification of effective resonance self-shielding cross sections, the efficient sampling and statistical analysis modules built in CUSA are used to quantify the uncertainty of eigenvalue propagated from multigroup microscopic cross sections. Also the "Sandwich" rule is used to quantify the uncertainty based on the calculated total sensitivity coefficients for comparison. The details and flowchart are nearly the same as those described in Section 3.1.

3.3. Model and Codes. In order to examine the performance of sensitivity and uncertainty analytical abilities of CUSA in the application of the uncertainty propagation and quantification of cross sections, a simple $\mathrm{UO}_{2}$ fuel pin cell is built, as shown in Figure 5. The density of $\mathrm{UO}_{2}$ is $10.3 \mathrm{~g} / \mathrm{cc}$, the enrichment of ${ }^{235} \mathrm{U}$ is $6.5 \%$ in this model, and the clad and moderator are Zr-nat and light water, respectively; the details of the materials can be found in Table 1 [28]. Here, it should be noted that the $\mathrm{Zr}$-nat is not treated as a resonance nuclide in our work.

As illustrated in Figure 5, the whole cell is divided into 12 subregions, in which the fuel zone is divided into 3 subregions and the moderator zone occupies 8 with flat-source approximation. It is due to the fact that the method of characteristics (MOC) is used and a home-developed Highfidelity NEutron Transport program for 3D nuclear reactor whole-core (HNET) is applied as a transport solver [29] in the resonance calculation and the following transport calculation. For the resonance calculation, a home-developed module built in the HNET based on the subgroup method is applied. Then a WIMS 69-energy group based library is used based on the ENDF/B VII.0 evaluation database [30]. At the same time, the multigroup covariance library ZZ-SCALE6.0/ COVA-44G has been used as the basic evaluated covariance information. At last, the CUSA is coupled with HNET to perform sensitivity and uncertainty analysis of effective resonance self-shielding cross sections and eigenvalue due to the microscopic cross sections.

\section{Numerical Results}

4.1. Sensitivity of Effective Resonance Self-Shielding XSs to MG Microscopic XSs. The sensitivity analysis of ${ }^{235} \mathrm{U}$ and ${ }^{238} \mathrm{U}$ effective resonance self-shielding cross sections to the multigroup microscopic cross sections of each nuclide, including resonance and nonresonant nuclides, is performed firstly. Figures 6-9 illustrate the top five most sensitive sources to the ${ }^{235} \mathrm{U}$ and ${ }^{238} \mathrm{U}$ effective resonance absorption and scattering cross section and the corresponding implicit sensitivity coefficients. 


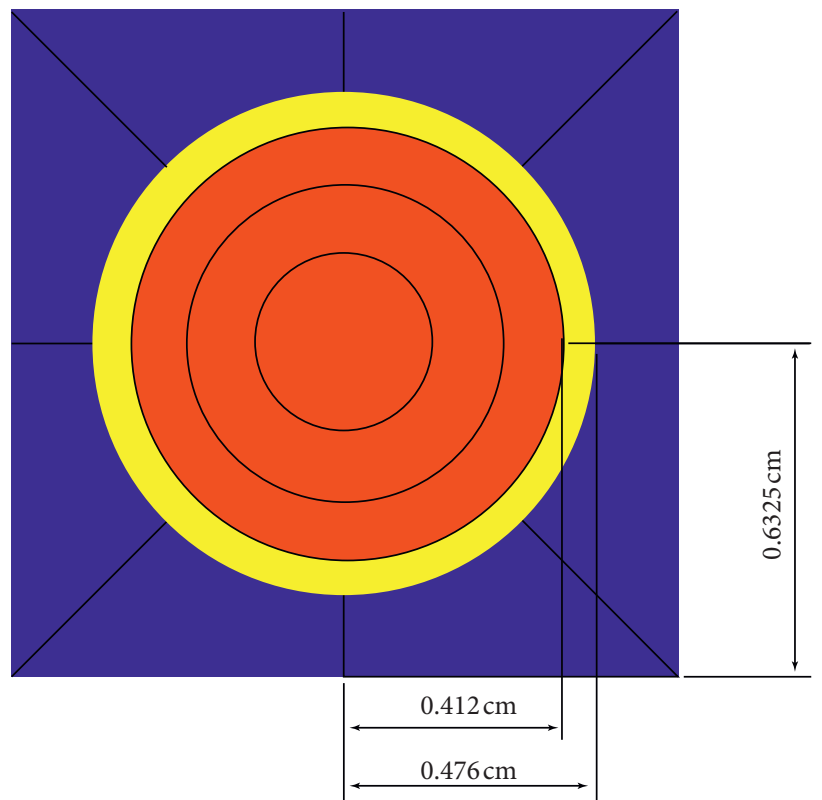

Figure 5: The simple $\mathrm{UO}_{2}$ fuel pin cell.

TABLE 1: The materials and the nuclide densities of the $\mathrm{UO}_{2}$ pin cell.

\begin{tabular}{|c|c|c|c|c|c|}
\hline \multirow{2}{*}{ Material } & \multicolumn{5}{|c|}{ Nuclide density $/\left(\mathrm{barn}^{-1} \cdot \mathrm{cm}^{-1}\right)$} \\
\hline & ${ }^{235} \mathrm{U}$ & ${ }^{238} \mathrm{U}$ & Zr-nat & ${ }^{1} \mathrm{H}$ & ${ }^{16} \mathrm{O}$ \\
\hline Fuel $(900 \mathrm{~K})$ & $1.5122 E-03$ & $2.1477 E-02$ & - & - & $4.5945 E-02$ \\
\hline Cladding $(600 \mathrm{~K})$ & - & - & $4.3107 E-02$ & - & - \\
\hline Moderator $(600 \mathrm{~K})$ & - & - & - & $4.4148 E-02$ & $2.2074 E-02$ \\
\hline
\end{tabular}

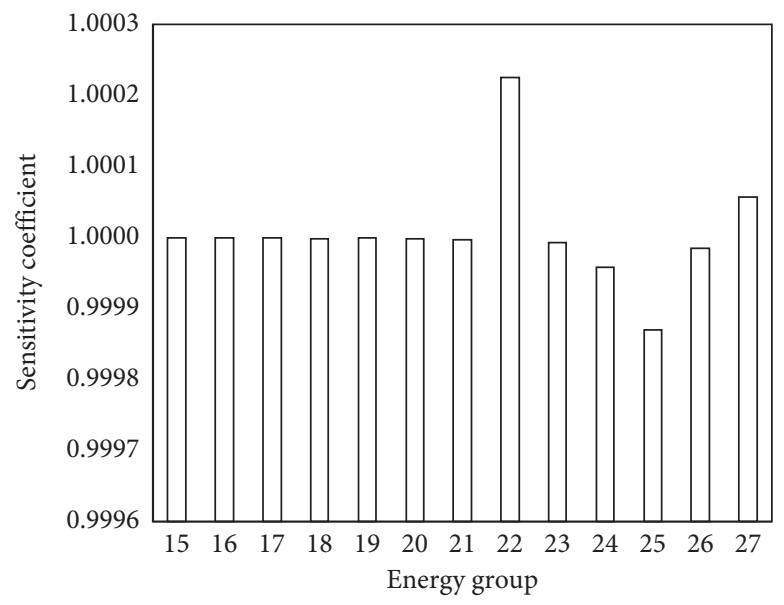

(a)

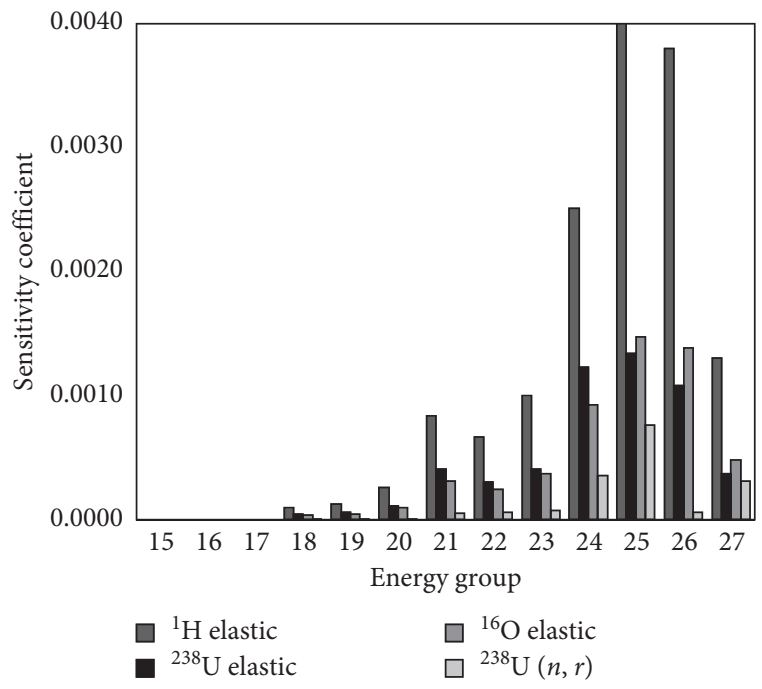

(b)

FIGURE 6: The top five most sensitive sources to the ${ }^{235} \mathrm{U}$ effective resonance scattering XS and the corresponding implicit sensitivity coefficients. (a) Sensitivity coefficient to ${ }^{235} \mathrm{U}$ elastic scattering. (b) Sensitivity coefficients to other four reactions.

It can be found that the effective resonance selfshielding cross section of each resonance nuclide is most sensitive to its associated multigroup microscopic cross section that constitutes this integral cross sections, as shown in (17), while the sensitivity coefficient to other microscopic cross sections is relatively small. For example, the effective resonance scattering cross sections of ${ }^{235} \mathrm{U}$ are quite sensitive to the associated microscopic elastic scattering cross section of ${ }^{235} \mathrm{U}$, the sensitivity coefficient of which is around 1 . But the second most sensitive source to 


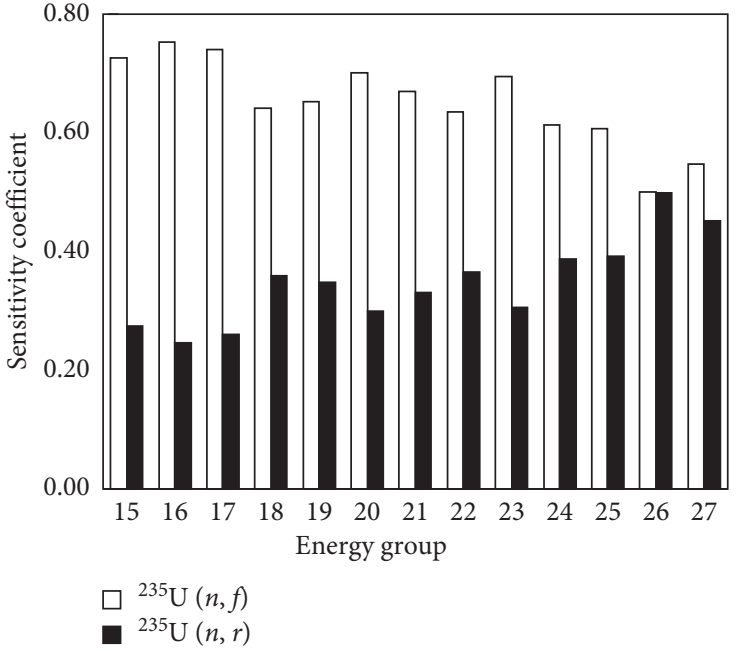

(a)

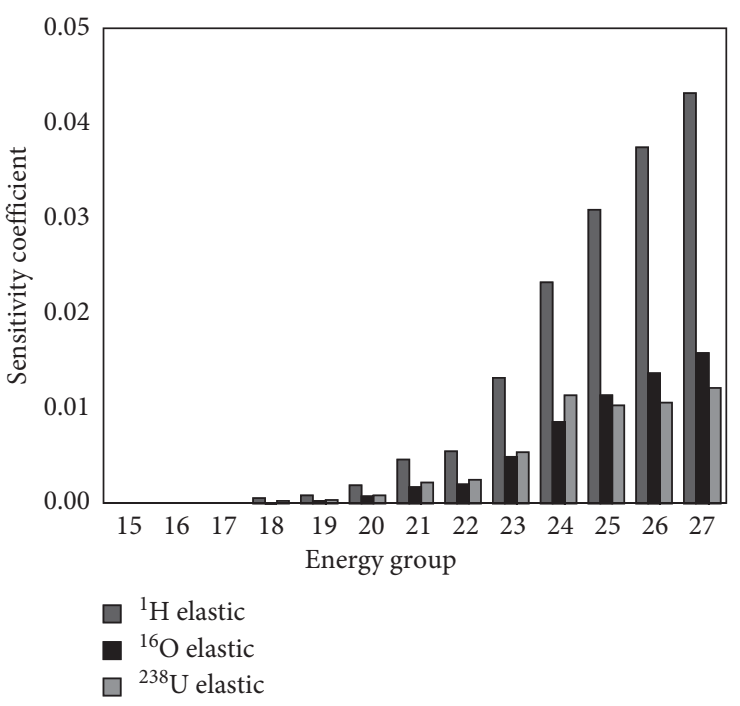

(b)

FIgURE 7: The top five most sensitive sources to the ${ }^{235} \mathrm{U}$ effective resonance absorption XS and the corresponding implicit sensitivity coefficients. (a) Sensitivity coefficients to ${ }^{235} \mathrm{U}$ fission and radiation capture. (b) Sensitivity coefficients to other three reactions.

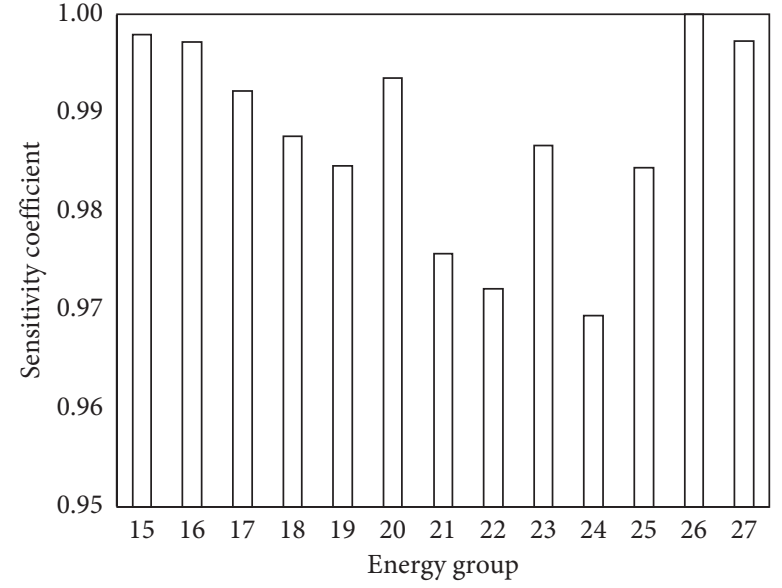

(a)

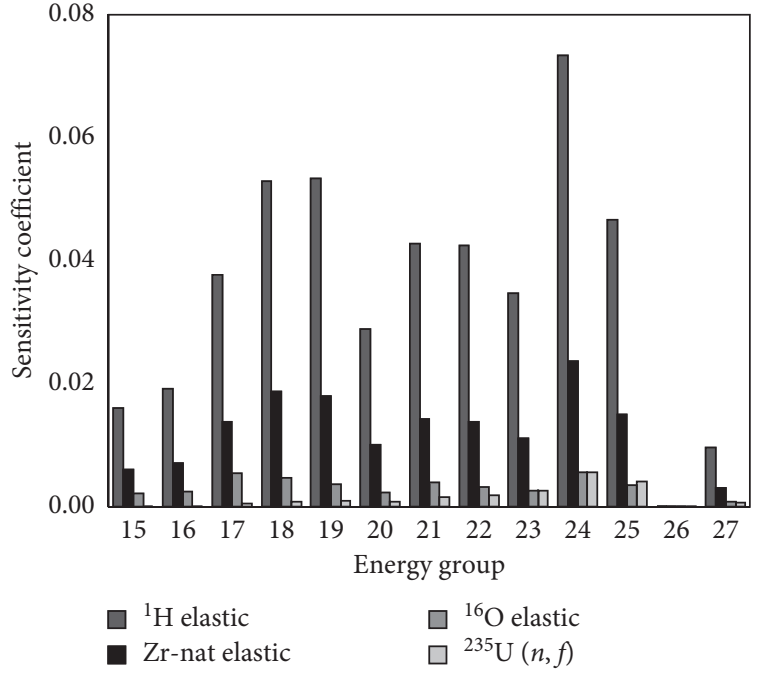

(b)

FIGURE 8: The top five most sensitive sources to the ${ }^{238} \mathrm{U}$ effective resonance scattering XS and the corresponding implicit sensitivity coefficients. (a) Sensitivity coefficient to ${ }^{238} \mathrm{U}$ elastic scattering. (b) Sensitivity coefficients to other four reactions.

the effective resonance scattering cross sections of ${ }^{235} \mathrm{U}$ is ${ }^{1} \mathrm{H}$ elastic scattering cross section, the value of which is only about 0.004 . Although the sensitivity coefficients of nonresonant nuclides are relatively small, these implicit effects cannot be ignored in the resonance calculation. The same as the scattering cross sections, the effective resonance absorption cross section is most sensitive to fission and radiation capture cross sections. For ${ }^{235} \mathrm{U}$, the top 1 most sensitive source is its fission reaction and the sensitivity coefficient is around 0.6 , which is followed by the radiation capture cross section of ${ }^{235} \mathrm{U}$ and the value is around 0.4 , while the top 1 most sensitive source is radiation capture reaction for ${ }^{238} \mathrm{U}$.
4.2. Uncertainty Quantification of Effective Resonance SelfShielding XSs. The size of sample space is crucial for nuclear cross-section uncertainty propagation and quantification by using statistical sampling methods. There are different sampling methods built in CUSA, such as SRS, LHS, IS, LHS-CDC, and LHS-SVDC. To determine a reasonable sample size for each sampling method, which is a relative small size for less computation burden and stable uncertainty contribution, the uncertainty contributions of radiation capture cross section to the $18^{\text {th }}$ and $22^{\text {nd }}$ group effective resonance absorption cross section of ${ }^{235} \mathrm{U}$ are selected and quantified by using different sampling methods with different sample size, as illustrated in Figure 10. At the 


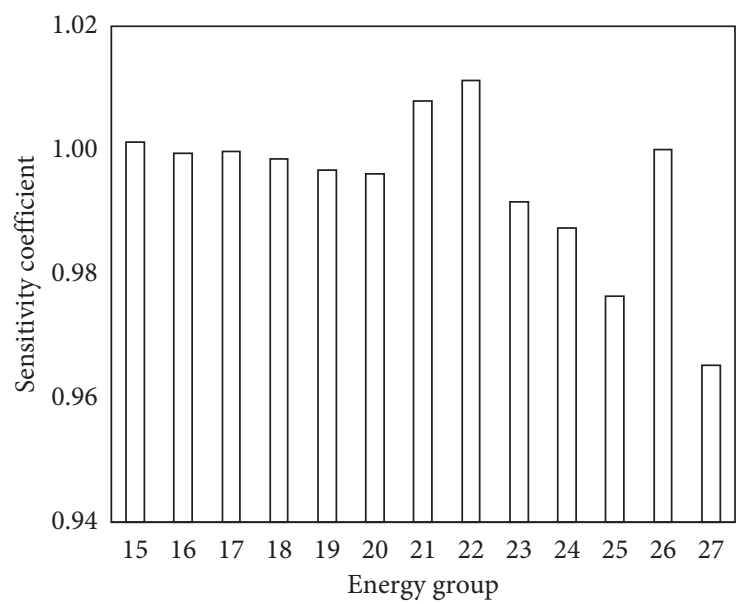

(a)

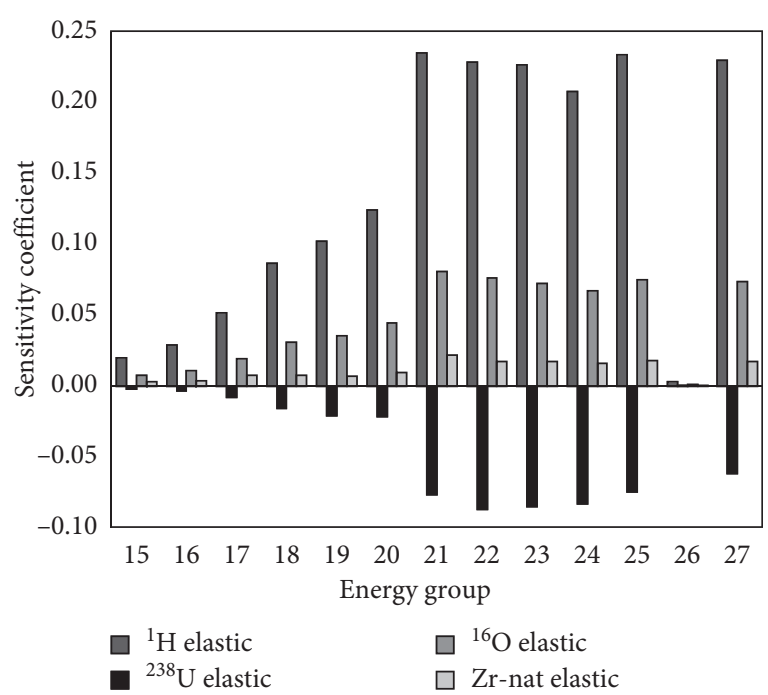

(b)

FIgURE 9: The top five most sensitive sources to the ${ }^{238} \mathrm{U}$ effective resonance absorption XS and the corresponding implicit sensitivity coefficients. (a) Sensitivity coefficient to ${ }^{238} \mathrm{U}$ radiation capture. (b) Sensitivity coefficients to other four reactions.

same time, the "Sandwich" rule is also used to quantify the uncertainty based on the calculated implicit sensitivity coefficients for comparison.

It is obviously found that the contribution of radiation capture cross section to the total uncertainty of the effective resonance absorption cross sections of ${ }^{235} \mathrm{U}$ tends to be stable and be close to the results quantified by using Sandwich rule when the sample size is greater than 100 for LHS-SVDC methods, while the sample size needs to be greater than 800 , which will generate a relatively stable uncertainty contribution for LHS or SRS methods. So the sample size is set to 100 and the LHS-SVDC built in CUSA is selected in the following uncertainty quantifications.

The relative uncertainty of the effective resonance absorption and scattering cross sections of ${ }^{235} \mathrm{U}$ and ${ }^{238} \mathrm{U}$ propagated from the multigroup microscopic cross sections is quantified by using CUSA and Sandwich rule, respectively, as shown in Figure 11. For uncertainty quantification, the error bar of the relative uncertainty under the sample size 100 is also quantified by using the statistical analytical function of CUSA, as illustrated in Figure 11. It is obviously found that the relative uncertainties quantified by CUSA agree well with the results calculated by the Sandwich rule based on the implicit sensitivity information obtained by CUSA. Therefore, the implicit sensitivity analysis model and the uncertainty quantification functions developed in CUSA can be proved correct and can be used for sensitivity and uncertainty analysis in nuclear reactor calculations.

Figure 11 also shows that the effective resonance absorption cross section of ${ }^{235} \mathrm{U}$ and the resonance scattering cross section of ${ }^{238} \mathrm{U}$ have a larger uncertainty due to the multigroup microscopic cross sections. The top 3 most significant microscopic cross section sources of uncertainty in these two effective resonance self-shielding cross sections are listed in Tables 2 and 3.
It can be seen that the relative uncertainties in the effective resonance self-shielding cross sections due to the basic microscopic cross sections are large and nonignorable. The neutron capture reaction of ${ }^{235} \mathrm{U}$ contributes the most to the uncertainty of ${ }^{235} \mathrm{U}$ resonance absorption cross section, up to $9 \%$, which is followed by ${ }^{235} \mathrm{U}$ fission reaction, while this effective resonance self-shielding cross section is most sensitive to the ${ }^{235} \mathrm{U}$ fission reaction. This difference is mainly attributed to the fact that the microscopic cross section of ${ }^{235} \mathrm{U}$ neutron capture reaction itself has a larger uncertainty than ${ }^{235} \mathrm{U}$ fission reaction. It can be also found that ${ }^{235} \mathrm{U}$ elastic scattering reaction has a larger contribution to uncertainty of the ${ }^{235} \mathrm{U}$ resonance absorption cross section than the ${ }^{1} \mathrm{H}$ elastic scattering reaction, although it is more sensitive to the ${ }^{1} \mathrm{H}$ elastic scattering reaction. This is also due to the fact that ${ }^{1} \mathrm{H}$ elastic scattering cross section has a smaller uncertainty. Similar phenomenon of ${ }^{1} \mathrm{H}$ elastic scattering reaction to the effective resonance scattering cross section of ${ }^{238} \mathrm{U}$ is also found. Although the effective resonance self-shielding cross sections are more sensitive to the ${ }^{1} \mathrm{H}$ elastic scattering reaction, while its contribution to uncertainty is small due to itself small uncertainty.

\subsection{Total Sensitivity Coefficient of Eigenvalue to MG Micro-} scopic XSs. The implicit and total sensitivity coefficients of $k_{\text {eff }}$ to the microscopic cross sections of each nuclide are calculated with the direct perturbation function of CUSA. The top four most sensitive sources to $k_{\text {eff }}$ and the corresponding implicit and total sensitivity coefficients are illustrated in Figure 12.

In our work, only the contribution of cross sections in resonance groups is investigated due to the fact that one of the main purposes is to verify the implicit sensitivity analytical ability of CUSA. It can be obviously found in Figure 12 that the total sensitivity is equal to the sum of explicit 


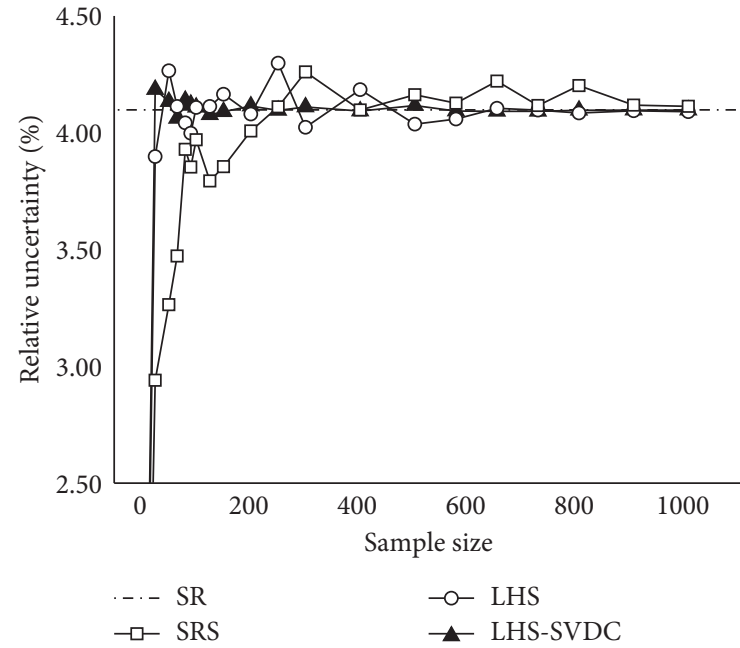

(a)

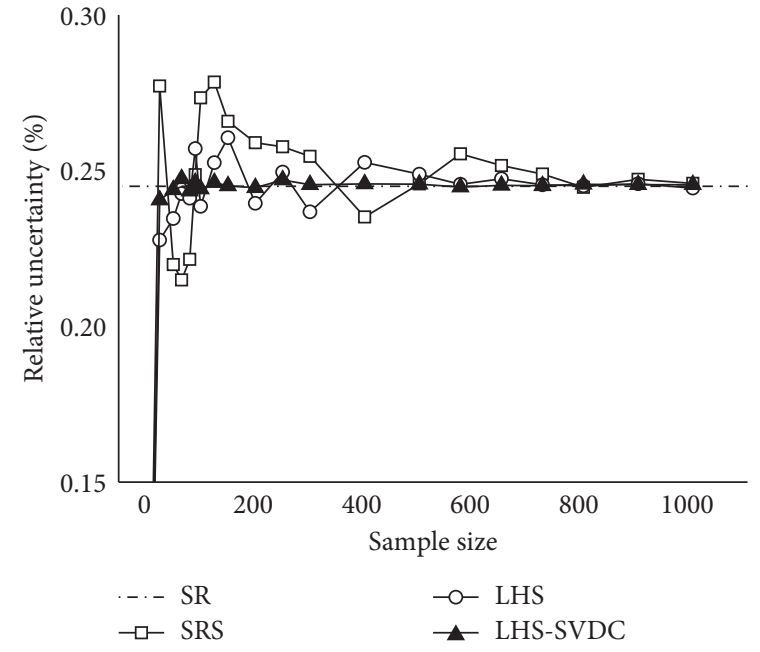

(b)

FIGURE 10: The contribution of ${ }^{235} \mathrm{U}(n, \gamma)$ to the uncertainty of ${ }^{235} \mathrm{U}$ resonance absorption XS by using different sampling methods with different sample sizes. (a) $18^{\text {th }}$ group. (b) $22^{\text {nd }}$ group.

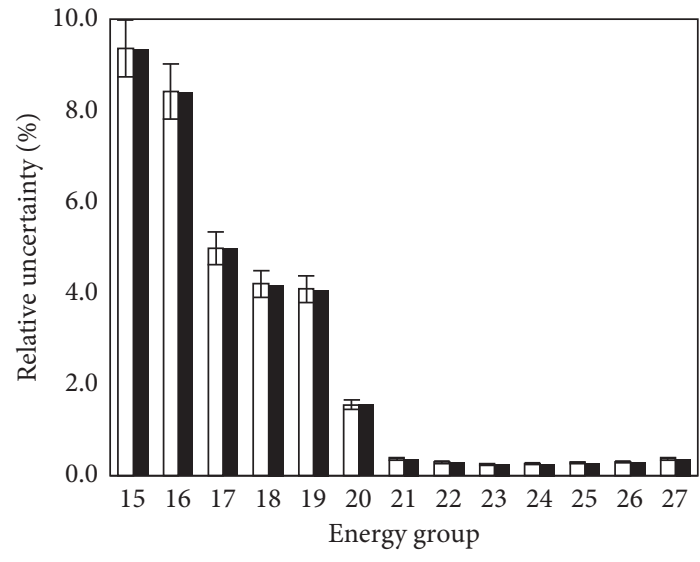

$\square \mathrm{SS}$

- SR

(a)

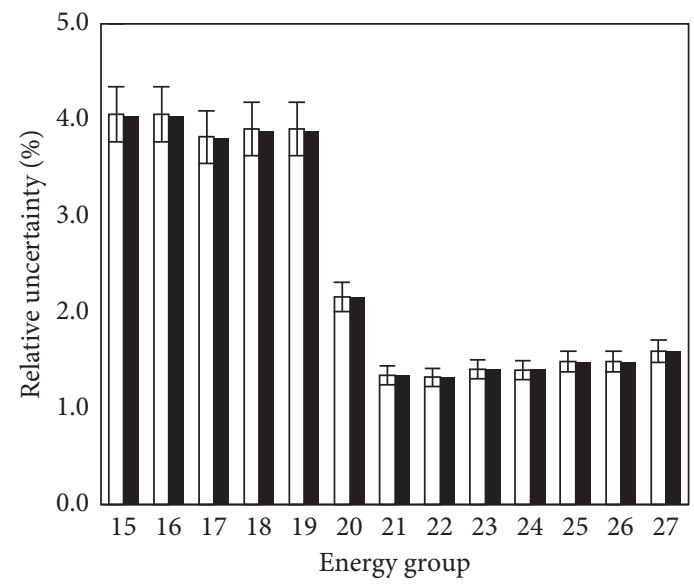

$\square$ SS

- SR

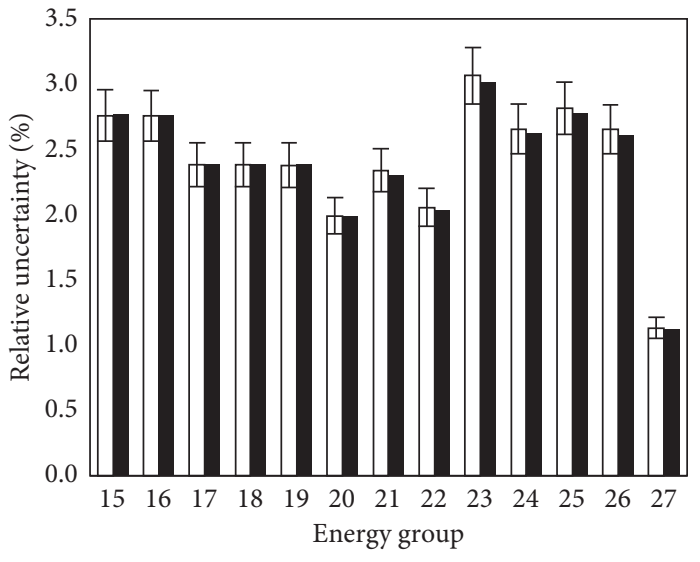

$\square$ SS

- $\mathrm{SR}$

(b)

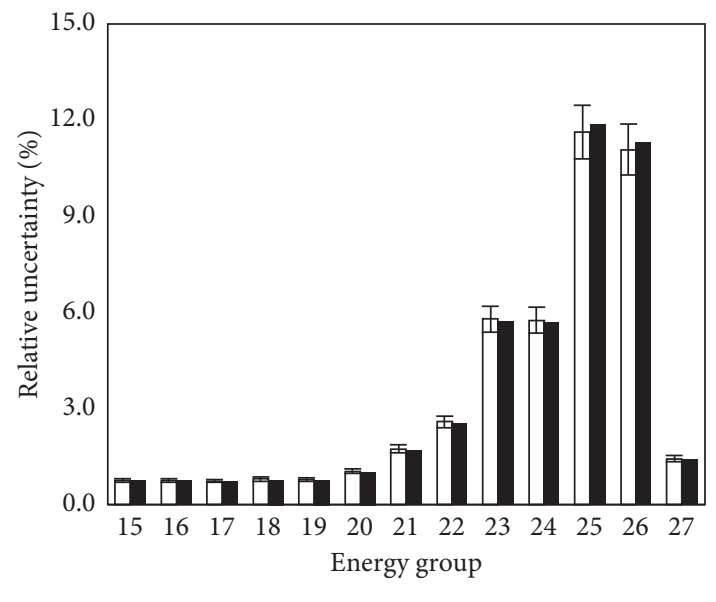

$\square$ SS

- SR

(c)

(d)

FIGURE 11: The uncertainty of effective resonance self-shielding cross sections. (a) ${ }^{235} \mathrm{U}$ effective resonance absorption XS. (b) ${ }^{238} \mathrm{U}$ effective resonance absorption XS. (c) ${ }^{235} \mathrm{U}$ effective resonance scattering XS. (d) ${ }^{238} \mathrm{U}$ effective resonance scattering XS. 
TABLE 2: The top 3 sources of uncertainty in ${ }^{235} \mathrm{U}$ effective resonance absorption XS.

\begin{tabular}{lccc}
\hline Energy group & ${ }^{235} \mathrm{U}(n, \gamma)$ & $\begin{array}{c}\text { Contributions to the relative uncertainty }(\%) \\
{ }^{235} \mathrm{U}(n, f)\end{array}$ & ${ }^{238} \mathrm{U}$ elastic \\
\hline 15 & $9.362 E+00 \pm 6.683 E-01$ & $3.441 E-01 \pm 2.435 E-02$ & $0.000 E+00$ \\
16 & $8.418 E+00 \pm 5.999 E-01$ & $3.572 E-01 \pm 2.528 E-02$ & $0.000 E+00$ \\
17 & $4.955 E+00 \pm 3.515 E-01$ & $6.125 E-01 \pm 4.334 E-02$ & $0.000 E+00$ \\
18 & $4.109 E+00 \pm 2.913 E-01$ & $9.056 E-01 \pm 6.409 E-02$ & $2.053 E-04 \pm 1.453 E-05$ \\
19 & $3.991 E+00 \pm 2.829 E-01$ & $9.201 E-01 \pm 6.512 E-02$ & $3.189 E-04 \pm 2.257 E-05$ \\
20 & $1.484 E+00 \pm 1.050 E-01$ & $4.932 E-01 \pm 3.490 E-02$ & $8.482 E-04 \pm 6.002 E-05$ \\
21 & $3.146 E-01 \pm 2.226 E-02$ & $2.101 E-01 \pm 1.487 E-02$ & $3.984 E-03 \pm 2.819 E-04$ \\
22 & $2.441 E-01 \pm 1.727 E-02$ & $1.790 E-01 \pm 1.266 E-02$ & $6.583 E-03 \pm 4.658 E-04$ \\
23 & $1.636 E-01 \pm 1.158 E-02$ & $1.974 E-01 \pm 1.397 E-02$ & $3.189 E-02 \pm 2.256 E-03$ \\
24 & $1.941 E-01 \pm 1.374 E-02$ & $1.675 E-01 \pm 1.186 E-02$ & $6.732 E-02 \pm 4.764 E-03$ \\
25 & $1.879 E-01 \pm 1.330 E-02$ & $1.835 E-01 \pm 1.299 E-02$ & $1.222 E-01 \pm 8.644 E-03$ \\
26 & $2.425 E-01 \pm 1.716 E-02$ & $1.496 E-01 \pm 1.059 E-02$ & $1.181 E-01 \pm 8.354 E-03$ \\
27 & $2.745 E-01 \pm 1.942 E-02$ & $2.664 E-01 \pm 1.885 E-02$ & $1.762 E-02 \pm 1.247 E-03$ \\
\hline
\end{tabular}

TABLE 3: The top 3 sources of uncertainty in ${ }^{238} \mathrm{U}$ effective resonance scattering XS.

\begin{tabular}{lccc}
\hline Energy group & ${ }^{238} \mathrm{U}$-elastic & $\begin{array}{c}\text { Contributions to the relative uncertainty }(\%) \\
16 \text { O-elastic }\end{array}$ & ${ }^{1} \mathrm{H}$ elastic \\
\hline 15 & $7.635 E-01 \pm 5.403 E-02$ & $1.145 E-02 \pm 8.101 E-04$ & $4.243 E-03 \pm 3.003 E-04$ \\
16 & $7.630 E-01 \pm 5.399 E-02$ & $1.343 E-02 \pm 9.500 E-04$ & $5.059 E-03 \pm 3.580 E-04$ \\
17 & $7.428 E-01 \pm 5.256 E-02$ & $1.324 E-02 \pm 9.369 E-04$ & $1.145 E-02 \pm 8.106 E-04$ \\
18 & $8.007 E-01 \pm 5.666 E-02$ & $1.831 E-02 \pm 1.296 E-03$ & $2.265 E-02 \pm 1.603 E-03$ \\
19 & $7.983 E-01 \pm 5.649 E-02$ & $1.767 E-02 \pm 1.250 E-03$ & $2.285 E-02 \pm 1.617 E-03$ \\
20 & $1.043 E+00 \pm 7.378 E-02$ & $9.930 E-03 \pm 7.026 E-04$ & $6.816 E-03 \pm 4.823 E-04$ \\
21 & $1.739 E+00 \pm 1.231 E-01$ & $1.395 E-02 \pm 9.872 E-04$ & $4.402 E-03 \pm 3.115 E-04$ \\
22 & $2.586 E+00 \pm 1.831 E-01$ & $1.345 E-02 \pm 9.520 E-04$ & $4.375 E-03 \pm 3.096 E-04$ \\
23 & $5.790 E+00 \pm 4.111 E-01$ & $1.086 E-02 \pm 7.685 E-04$ & $3.582 E-03 \pm 2.534 E-04$ \\
24 & $5.756 E+00 \pm 4.086 E-01$ & $2.316 E-02 \pm 1.639 E-03$ & $7.555 E-03 \pm 5.346 E-04$ \\
25 & $1.162 E+01 \pm 8.337 E-01$ & $1.464 E-02 \pm 1.036 E-03$ & $4.805 E-03 \pm 3.400 E-04$ \\
26 & $1.108 E+01 \pm 7.938 E-01$ & $2.401 E-05 \pm 1.699 E-06$ & $6.803 E-06 \pm 4.814 E-07$ \\
27 & $1.439 E+00 \pm 1.019 E-01$ & $2.993 E-03 \pm 2.118 E-04$ & $9.874 E-04 \pm 6.987 E-05$ \\
\hline
\end{tabular}

and implicit sensitivity, which further verifies the reliability of implicit and total sensitivity analysis abilities of CUSA. At the same time, the $k_{\text {eff }}$ total sensitivity coefficient to the neutron radiation capture reaction of ${ }^{235} \mathrm{U}$ and ${ }^{238} \mathrm{U}$ is negative and is positive for the fission reaction of ${ }^{235} \mathrm{U}$ and elastic scattering reaction of ${ }^{1} \mathrm{H}$. This is physically consistent with the fact that the radiation capture reaction results in the disappearance of neutron, while fission results in the production of neutron and the moderation produces more thermal neutron, which has a positive effect on the fission event.

As discussed in Section 3.2, the explicit effect is considered when calculating the implicit sensitivity coefficient for resonance nuclides, as shown in Figures 12(a)-12(c). But for the nonresonant nuclides, the explicit and implicit sensitivity coefficients should be calculated, respectively. It can be found that the explicit sensitivity coefficients of most energy group for nonresonant nuclides are positive, while the implicit sensitivity coefficients are negative. It is worth noting that the total and implicit sensitivity coefficients of ${ }^{1} \mathrm{H}$ are the biggest one compared with other nonresonant nuclides due to the fact that the ${ }^{1} \mathrm{H}$ is the dominating moderated nuclide in our studies.
4.4. Uncertainty Quantification of Eigenvalue due to $M G$ Microscopic XSs. The contributions of the multigroup microscopic cross sections in resonance groups to the $k_{\text {eff }}$ uncertainty and the total relative uncertainty of $k_{\text {eff }}$ propagated from these cross sections are quantified by using CUSA and Sandwich rule, respectively, as presented in Table 4. It is obviously found that the contributions and the relative uncertainties quantified by CUSA agree well with the results calculated by the Sandwich rule.

It can be found that the relative uncertainty in $k_{\text {eff }}$ due to the basic cross section in resonance group is large and nonignorable, of which the neutron capture reaction of ${ }^{238} \mathrm{U}$ contributes the most to the $k_{\text {eff }}$ uncertainty, up to $0.27 \%$. And $k_{\text {eff }}$ is also most sensitive to the neutron capture reaction of ${ }^{238} \mathrm{U}$ in resonance region, while it is most sensitive to the average number of neutrons emitted per fission event for all energy regions. Besides, although $k_{\text {eff }}$ is more sensitive to the ${ }^{1} \mathrm{H}$ elastic scattering reaction than the neutron capture reaction of ${ }^{235} \mathrm{U}$, its associated contribution to the $k_{\text {eff }}$ uncertainty is smaller due to itself smaller uncertainty. The whole relative uncertainty of $k_{\text {eff }}$ contributed by resonance energy groups is about $0.3 \%$ and it is reasonable that the value is smaller than the standard value 


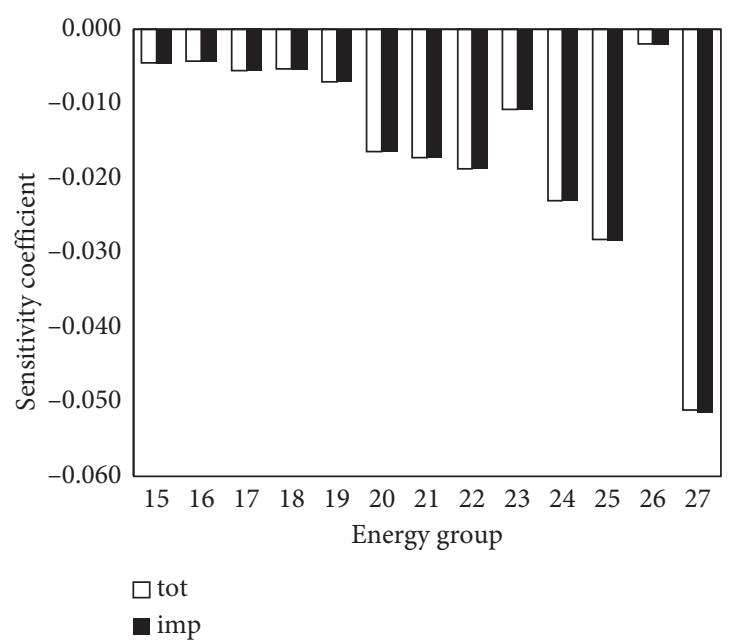

(a)

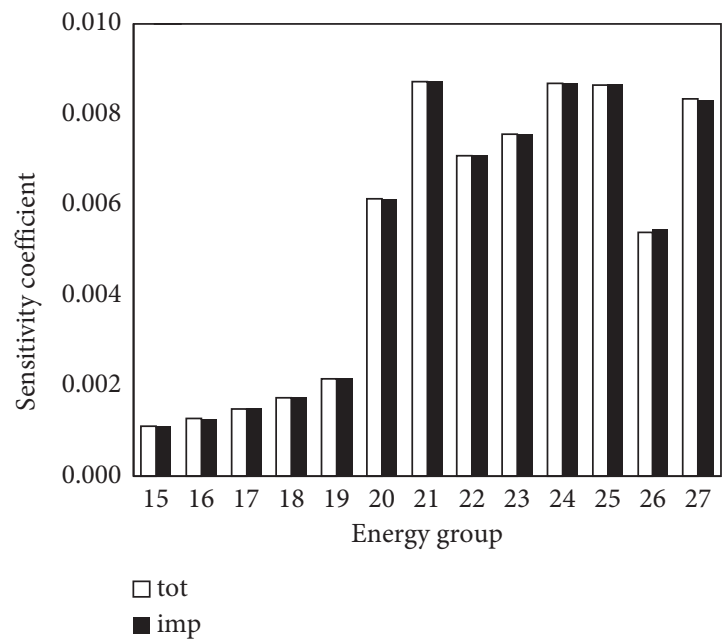

(c)

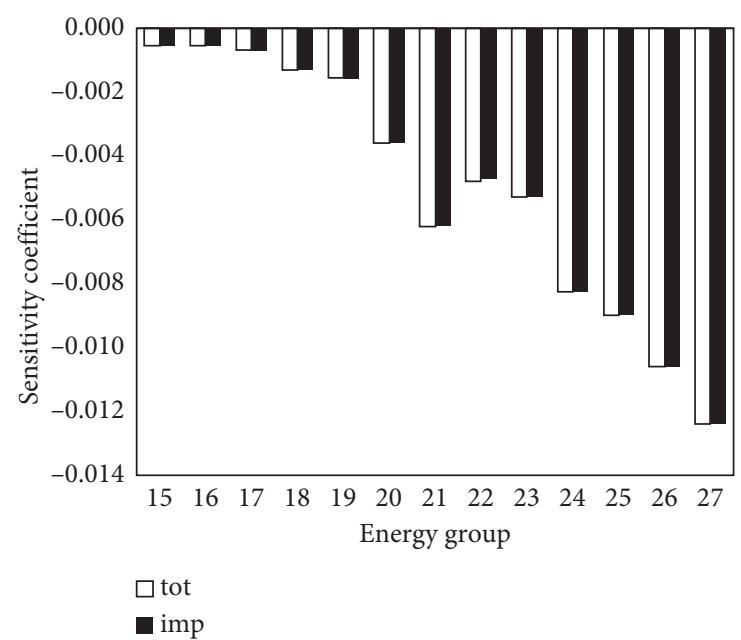

(b)

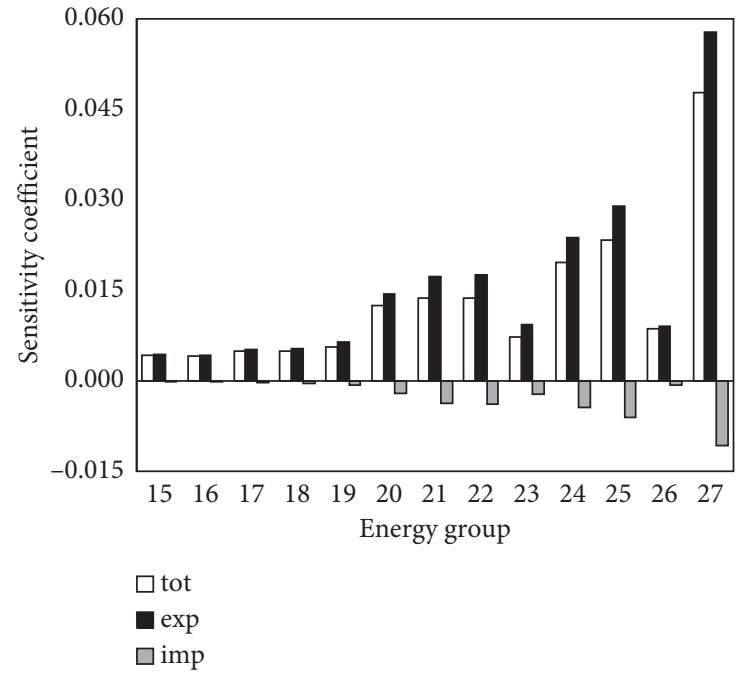

(d)

FIGURE 12: The total and implicit sensitivity coefficients of $k_{\text {eff }}$ to the microscopic cross sections. (a) ${ }^{238} \mathrm{U}(n, \gamma)$. (b) ${ }^{235} \mathrm{U}(n, \gamma)$. (c) ${ }^{235} \mathrm{U}(n, f)$. (d) ${ }^{1} \mathrm{H}$ elastic scattering.

TABLE 4: The contributions of microscopic cross sections to the $k_{\text {eff }}$ uncertainty.

\begin{tabular}{lcrl}
\hline Cross section type & Total sensitivity coefficient & Uncertainty (\%) (sandwich rule) & $\begin{array}{c}\text { Uncertainty }(\%) \\
\text { Statistics sampling }\end{array}$ \\
\hline${ }^{238} \mathrm{U}(n, \gamma)$ & $-1.948 E-01$ & $2.692 E-01$ & $2.692 E-01 \pm 1.905 E-02$ \\
${ }^{235} \mathrm{U}(n, \gamma)$ & $-6.472 E-02$ & $1.007 E-01$ & $1.012 E-01 \pm 7.161 E-03$ \\
$\mathrm{Zr}-$ nat $(n, \gamma)$ & $-5.147 E-03$ & $5.988 E-02$ & $6.036 E-02 \pm 4.271 E-03$ \\
${ }^{235} \mathrm{U}(n, f)$ & $6.838 E-02$ & $2.026 E-02$ & $2.032 E-02 \pm 1.438 E-03$ \\
${ }^{1} \mathrm{H}$ elastic & $1.707 E-01$ & $1.979 E-02$ & $2.045 E-02 \pm 1.447 E-03$ \\
${ }^{238} \mathrm{U}$-elastic & $4.089 E-03$ & $9.781 E-03$ & $9.709 E-03 \pm 6.870 E-04$ \\
${ }^{16} \mathrm{O}$-elastic & $-9.390 E-03$ & $9.327 E-03$ & $9.225 E-03 \pm 6.549 E-06$ \\
${ }^{235} \mathrm{U}$ elastic & $-2.915 E-04$ & $1.380 E-03$ & $1.384 E-03 \pm 9.793 E-05$ \\
$\mathrm{Zr}-$ nat-elastic & $-2.107 E-03$ & $1.305 E-03$ & $1.320 E-03 \pm 9.341 E-05$ \\
${ }^{16} \mathrm{O}(n, \gamma)$ & $-1.013 E-06$ & $9.880 E-06$ & $9.725 E-06 \pm 6.881 E-07$ \\
${ }^{1} \mathrm{H}(n, \gamma)$ & $-1.636 E-03$ & $8.159 E-04$ & $8.262 E-04 \pm 5.846 E-05$ \\
Sum & - & $2.953 E-01$ & $2.956 E-01 \pm 2.091 E-02$ \\
\hline
\end{tabular}


(0.5\%) because fast and thermal energy groups are not in consideration.

\section{Conclusions}

Best-Estimation Plus Uncertainty (BEPU) analysis method can provide more information to improve the reliability of calculation results than the safety analysis with conservative assumption. And the statistical sampling-based uncertainty and sensitivity analysis methods are widely used in practical applications of the multiphysics, multiscale coupling nuclear reactor system. In this paper, a novel and efficient sampling method for inputs with normal and uniform distributions is introduced, which is based on the well-known LHS method and SVD. The efficient sampling method based on the SVD for high-dimensional covariance matrix is also proposed. Then, a systematic theory for uncertainty and sensitivity analysis is established based on the classical statistical theory, which can be used to quantify the distribution type of outputs, uncertainty, and its associated error bar under a specific size of samples. Based on these new strategies, the CUSA has been updated. At the same time, a user-friendly interface for CUSA has been developed and the interface also serves as an integrated platform for controlling all the function modules and coupling with the executable version of other simulation codes.

For applications, the uncertainties of effective resonance self-shielding cross sections and total uncertainty of eigenvalue propagated from multigroup microscopic cross sections are quantified by using the CUSA and a homedeveloped resonance calculation code. Especially, the explicit and implicit effects for resonance and nonresonance isotopes are fully considered. The calculations of total sensitivity coefficient for the resonant nuclides and nonresonant nuclides are slightly different. For the resonant nuclides, the explicit sensitivity coefficient term should be considered as zero. This is due to the fact that the explicit effect is already considered when calculating the implicit sensitivity coefficients for resonant nuclides. But for the nonresonant nuclides, since there is no effective resonance self-shielding cross section associated with its own multigroup microscopic cross section, the explicit effect is not considered in the process of quantifying its implicit effect on the effective resonance self-shielding cross sections of resonant nuclides. Finally, a simple $\mathrm{UO}_{2}$ pin cell is considered to examine the performance of CUSA and the total uncertainty and sensitivity analysis ability.

The numerical results indicate that the relative uncertainties quantified by CUSA agree well with the results calculated by the Sandwich rule based on the implicit sensitivity information obtained by CUSA. Therefore, the implicit sensitivity analysis model and the uncertainty quantification functions developed in CUSA can be proved correct and can be used for sensitivity and uncertainty analysis in nuclear reactor calculations. Moreover, the LHS-SVDC is recommended to propagate the uncertainty in multigroup cross sections, and then a more reliable uncertainty prediction with smaller number of samples can be obtained.
However, there is still some work to be solved and improved in the further work. On the one hand, the benchmark needs to be replaced with a more representative one, since the enrichment of ${ }^{235} \mathrm{U}$ (6.5\%) exceeded the standard of LWR. On the other hand, it is necessary to compare the calculation results of CUSA with other advanced solvers such as TSUNAMI and Sampler. Finally, it makes sense to apply CUSA to other parts of nuclear reactor calculations such as thermal hydraulic calculation.

\section{Data Availability}

The data used to support the findings of this study are available from the corresponding author upon request.

\section{Conflicts of Interest}

The authors declare that they have no conflicts of interest.

\section{Acknowledgments}

This work was supported by the National Key R\&D Program of China (2018YFE0180900).

\section{References}

[1] D. R. Gaston, C. J. Permann, J. W. Peterson et al., "Physicsbased multiscale coupling for full core nuclear reactor simulation," Annals of Nuclear Energy, vol. 84, pp. 45-54, 2015.

[2] R. N. Bratton, M. Avramova, and K. Ivanov, "Oecd/nea benchmark for uncertainty analysis in modeling (Uam) for LWRS-summary and discussion of neutronics cases (Phase I)," Nuclear Engineering and Technology, vol. 46, no. 3, pp. 313-342, 2014.

[3] F. D'Auria, H. Glaeser, and S. Lee, "Best estimate safety analysis for nuclear power plants: uncertainty evaluation," IAEA Safety Report Series, IAEA, Vienna, Austria, 2008.

[4] A. Yamamoto, Y. Yasue, T. Endo, Y. Kodama, and M. Tatsumi, "Uncertainty estimation of core safety parameters using cross-correlations of covariance matrix," Journal of Nuclear Science and Technology, vol. 50, no. 10, pp. 966-978, 2013.

[5] M. L. Williams, "Perturbation theory for nuclear reactor analysis," CRC Handbook of Nuclear Reactors Calculations, vol. 3, pp. 63-188, 1986.

[6] J. C. Helton, J. D. Johnson, C. J. Sallaberry, and C. B. Storlie, "Survey of sampling-based methods for uncertainty and sensitivity analysis," Reliability Engineering \& System Safety, vol. 91, no. 10-11, pp. 1175-1209, 2006.

[7] H. Yun, K. Park, W. choi, and S. G. Hong, "An efficient evaluation of depletion uncertainty for a GBC-32 dry storage cask with PLUS7 fuel assemblies using the Monte Carlo uncertainty sampling method," Annals of Nuclear Energy, vol. 110, pp. 679-691, 2017.

[8] Z. Choi, L. Cao, H. Wu, W. Shen, Y. Liu, and C. Wan, "Sensitivity and uncertainty analysis for the PWR online power-distribution monitoring with NECP-ONION system," Annals of Nuclear Energy, vol. 114, pp. 359-368, 2018.

[9] G. Ilas and H. Liljenfeldt, "Decay heat uncertainty for BWR used fuel due to modeling and nuclear data uncertainties," Nuclear Engineering and Design, vol. 319, pp. 176-184, 2017.

[10] C. Hao and P. Li, "A new efficient sampling method for quantifying and propagating nuclear data uncertainty in 
CUSA," Nuclear Safety and Simulation, vol. 8, no. 4, pp. 325-332, 2018.

[11] C. Hu, F. Li, G. Jiong, and W. Lidong, "Uncertainty and sensitivity analysis of filling fraction of pebble bed in pebble bed HTR," Nuclear Engineering and Design, vol. 292, pp. 123-132, 2015.

[12] M. Kloos and E. Hofer, "SUSA-PC: a personal computer version of the program system for uncertainty and sensitivity analysis of results from computer models, version 3.2, user's guide and tutorial," Gesellschaft für Anlagen und Reaktorsicherheit, 1999.

[13] W. Zwermann, A. Aures, L. Gallner et al., "Nuclear data uncertainty and sensitivity analysis with XSUSA for fuel assembly depletion calculations," Nuclear Engineering and Technology, vol. 46, no. 3, pp. 343-352, 2014.

[14] M. S. Eldred, K. R. Dalbey, W. J. Bohnhoff et al., "Dakota: a multilevel parallel object-oriented framework for design optimization, parameter estimation, uncertainty quantification, and sensitivity analysis: version 5.0, user's manual," Office of Scientific \& Technical Information, Technical Reports, Oak Ridge, TN, USA, 2010.

[15] C. Hao, "Uncertainty analysis in modelling of pebble bed HTR," Doctoral thesis, Institute of Nuclear and New Energy Technology, Beijing China, 2014.

[16] A. Yamamoto, K. Kinoshita, T. Watanabe et al., "Uncertainty quantification of LWR core characteristics using random sampling method," Nuclear Science and Engineering, vol. 181, no. 2, pp. 160-174, 2015.

[17] R. J. A. Tough and K. D. Ward, “The correlation properties of gamma and other non-Gaussian processes generated by memoryless nonlinear transformation," Journal of Physics D: Applied Physics, vol. 32, no. 23, pp. 3075-3084, 1999.

[18] C. Hao, F. Li, W. Hu, Y. Zhang, and Q. Zhao, "Quantification of control rod worth uncertainties propagated from nuclear data via a hybrid high-order perturbation and efficient sampling method," Annals of Nuclear Energy, vol. 114, pp. 227-235, 2018.

[19] J. Frank and Massey Jr., "The Kolmogorov-Smirnov test for goodness of fit," Journal of the American Statistical Association, vol. 46, no. 253, pp. 68-78, 1951.

[20] B. L. van der Waerden, Mathematical Statistics, Intext Educational Publishers, New York, NY, USA, 1971.

[21] A. Sangtae and J. A. Fessler, Standard Errors of Mean, Variance, and Standard Deviation Estimators, The University of Michigan, Ann Arbor, MI, USA, 2003.

[22] G. S. Chen, "Interval estimate of the interval length on uniform distribution," Pure and Applied Mathematics, vol. 3, pp. 63-68, 2006.

[23] T. Zhu, A. Vasiliev, H. Ferroukhi, D. Rochman, and A. Rochman Pautz, "Testing the Sampling-Based NUSS-RF Tool for the Nuclear Data-Related Global Sensitivity Analysis with Monte Carlo Neutronics Calculations," Nuclear Science and Engineering, vol. 184, no. 1, pp. 69-83, 2016.

[24] T. A. Mara and S. Tarantola, "Variance-based sensitivity indices for models with dependent inputs," Reliability Engineering \& System Safety, vol. 107, pp. 115-121, 2012.

[25] Q. Zhao, C. Zhang, C. Hao, F. Li, D. Wang, and Y. Yu, "New strategies for quantifying and propagating nuclear data uncertainty in CUSA," Nuclear Engineering and Design, vol. 307, pp. 328-338, 2016.

[26] R. MacFarlane and D. Muir, The NJOY Nuclear Data Processing System Version91, Los Alamos National Laboratory, Los Alamos, NM, USA, 1994.
[27] NEA Data Bank, "ZZ-SCALE6.0/COVA-44G, a 44-group cross section covariance matrix library retrieved from the SCALE 6.0 package," Radiation Safety Information Computational Center at Oak Ridge National Laboratory, Oak Ridge, TN, USA, 2011.

[28] A. Yamamoto, T. Ikehara, T. Ito, and E. Saji, "Benchmark problem suite for reactor physics study of LWR next generation fuels," Journal of Nuclear Science and Technology, vol. 39, no. 8, pp. 900-912, 2002.

[29] C. Ito, L. Kang, Y., P. Song, Q. Zhao, and Z. Zhang, “3D whole-core neutron transport simulation using 2D/1D method via multi-level generalized equivalence theory based CMFD acceleration," Annals of Nuclear Energy, vol. 122, pp. 79-90, 2018.

[30] M. B. Chadwick, P. Obložinský, M. Herman et al., "ENDF/BVII.0: next generation evaluated nuclear data library for nuclear science and technology," Nuclear Data Sheets, vol. 107, no. 12, pp. 2931-3060, 2006. 